EmRe SelcuK, VIVIAN Zayas, AND Cindy HaZAN Cornell University

\title{
Beyond Satisfaction: The Role of Attachment in Marital Functioning
}

In this article, we investigate marital functioning from an attachment theory perspective. We review empirical evidence showing that, in nondistressed marriages, spouses, as attachment figures, provide each other with a subjective sense of felt security, regulate each other's affective and physiological states, and facilitate each other's functioning outside the relationship, especially with respect to exploratory behaviors. These provisions of the attachment bond occur independent of the level of satisfaction experienced in the marriage and may even occur when the marital relationship itself is not very satisfying. In addition, we discuss the role of attachment style in marital functioning. We conclude by suggesting future directions in which an attachment theory perspective may complement existing theory and research in marital relations.

Attachment seems to develop regularly in marriage so long as the spouse is adequately accessible and attentive.... Once developed, attachment seems to persist. Even when marriages turn bad and the other components of love fade or turn into their opposites, attachment is likely to remain.

-Weiss, 1975, p. 44

Imagine two married couples. Jamie and Pat are very satisfied with their relationship. They

Department of Human Development, Cornell University, G94 Martha van Rensselaer Hall, Ithaca, NY 14853 (es588@cornell.edu).

Key Words: attachment, exploration, felt security, marital relationships, stress buffering, synchrony. love each other deeply. They share many activities and spend a lot of time together. When something bad happens, they turn to each other. They feel secure in each other's presence and uneasy when either is away for an occasional trip. Kris and Terry, in contrast, are no longer very happy with their relationship. They do not spend much time together or share intimate moments as they used to do in the past. When they both are at home, they spend most of their time in separate rooms and interact very little. Nevertheless, when things go wrong, they immediately think of each other. They cannot stand the idea of divorce. They want each other in the next room, not further away.

Marital researchers have traditionally focused on marital quality, assessed with measures of adjustment, happiness, and most commonly satisfaction (Bradbury, Fincham, \& Beach, 2000; Gottman, 1982): What are the factors contributing to Jamie and Pat's satisfaction with their relationship? Why aren't Kris and Terry happy with theirs? Answering these questions is important for many reasons. For example, marital satisfaction is critical to the well-being of spouses (e.g., Saxbe, Repetti, \& Nishina, 2008) and their children (Gable, Belsky, \& Crnic, 1992). Happy marriages also have been associated with benefits to society, such as a decreased likelihood of committing crimes (Sampson, Laub, \& Wimer, 2006). In addition, marital satisfaction is an important predictor of marital stability (Karney \& Bradbury, 1995), and research on its antecedents is instrumental for developing successful therapeutic interventions aimed to prevent divorce. 
Although research on marital quality has immense basic and applied value, the almostexclusive focus on satisfaction minimizes, and often completely ignores, the role of attachment in marriage. In contrast to a focus on marital satisfaction, an attachment researcher might ask: What is the nature of the bond between Jamie and Pat, and Kris and Terry? How does it affect their physical and psychological functioning? Despite differences in satisfaction, does the attachment bond serve similar functions in the two couples' relationships, or is it likely to differ? An attachment theory (Bowlby, 1973, 1980, 1982) perspective may complement the marital literature by offering answers to those questions.

Although attachment theory was initially developed to explain the complex nature of infant-caregiver relationships, the theory was extended to understand the emotional ties between adult romantic partners (e.g., Hazan \& Shaver, 1987, 1990). For more than two decades, attachment theory has been one of the most widely used theories for studying adult romantic relationships (Cassidy \& Shaver, 2008; Mikulincer \& Shaver, 2007). Yet there has been surprisingly little cross-fertilization between researchers interested in marital relations and those interested in adult romantic attachment.

In this article, we seek to highlight the utility of applying an attachment theory perspective to the study of marital relationships as a complement to current perspectives focusing on marital satisfaction. We propose that the tie between spouses possesses all the features that characterize an attachment bond. As such, spouses' physical and psychological functioning, both within and outside the relationship, is enhanced by the maintenance of this bond and compromised by its disruption. Moreover, we argue that the provisions of the attachment bond in marriage are likely to occur independent of the level of marital satisfaction and may even occur when the marital relationship is not highly satisfying. Direct empirical evidence for this argument is still scant. Studies have yet to investigate attachment processes specifically in marital relationships with very low levels of satisfaction. Moreover, even among the existing studies that focus on moderate to highly satisfied couples, only a few have investigated the role of marital quality on attachment processes. Despite the need for further research recruiting samples with a broader range of marital satisfaction, the extant findings, as we will review, provide support for the idea that attachment confers psychological and physical benefits, above and beyond those conferred by marital satisfaction.

In the sections that follow, we first identify the basic features and functions of attachment bonds by providing a brief overview of central concepts in infant and adult attachment theory. Then, we discuss three ways in which attachment theory speaks to marital relations. We conclude by discussing future directions for research and theory on marital relationships that incorporates an attachment perspective.

\section{ATTACHMENT IN INFANCY AND ADULTHOOD}

Bowlby (1982) argued that evolution equipped human beings with many behavioral systems, including attachment, caregiving, exploration, affiliation, and sexual mating systems, which promote an individual's survival and reproductive success. Each of these systems becomes activated or deactivated as a function of the individual's current psychological state and environmental context, and each has distinct but interrelated functions. In particular, the function of the attachment system is to ensure that in early life an infant maintains proximity to important others (attachment figures) who are able to provide protection, support, and comfort.

Attachment, however, is not limited to early life and "is held to characterize human beings from the cradle to the grave" (Bowlby, 1979, p. 129). The prototypical attachment relationship in infancy is between the infant and the parent, whereas the prototypical attachment relationship in adulthood is the pair bond (see also Ainsworth, 1991; Hazan \& Shaver, 1987, 1990; Weiss, 1975). Both in infancy and adulthood, the goal of the attachment system is the same: achieving a sense of protection by maintaining proximity to attachment figures. This sense of protection involves felt security (Sroufe \& Waters, 1977a), a psychological state characterized by feelings of safety and comfort, and the belief that the attachment figure is accessible and responsive.

Deriving felt security from attachment relationships, both in infancy and in adulthood, is associated with important implications for psychological and social functioning: According to the theory, changes in felt security determine the activation and deactivation of the attachment system. As long as the individual maintains a sense of security, the system is quiescent, 
enabling the individual to devote her resources to activities other than self-protection. In infancy, these activities involve playing and exploring the environment. In adulthood, work and leisure activities are prominent examples of exploration (e.g., Hazan \& Shaver, 1990). Appraising the external environment as threatening, the self as in need of help (e.g., due to pain or sickness), or the attachment figure as inaccessible and unresponsive decreases felt security and activates the attachment system. As a result, the individual seeks proximity to attachment figures (e.g., Collins \& B. C. Feeney, 2000; Fraley \& Shaver, 1998; Mikulincer, Birnbaum, Woddis, \& Nachmias, 2000), ceasing exploratory behavior (e.g., Ainsworth, Blehar, Waters, \& Wall, 1978). In infancy, these proximity bids (e.g., crying, calling out or moving toward the parent) aim to establish actual contact with the attachment figure (Ainsworth et al., 1978). In adulthood, symbolic contact via calling the mental representation of the attachment figure into mind (Mikulincer \& Shaver, 2004; Uvnäs-Moberg, 1998) may be sufficient to regain at least some felt security, although the individual may still need actual contact in case of severe stressors. In both early and adult life, contact with the attachment figure restores felt security and deactivates the attachment system. As a result, the individual resumes exploratory activities.

Individuals form many relationships with others throughout their life, and not all close relationships are necessarily attachments. An attachment relationship is assumed to exist if the individual selectively directs four classes of behavior to a close relationship partner: The individual (a) maintains proximity to the relationship partner, (b) retreats to the relationship partner as a safe haven when threatened or in need of comfort, (c) uses the relationship partner as a secure base from which to explore the world, and (d) experiences separation distress when the relationship partner is not accessible. These behaviors are readily observed in 1-year-old infants' interactions with their parents. In adulthood, they are directed toward romantic partners, close friends, and adult children, in addition to parents (Doherty \& Feeney, 2004; Fraley \& Davis, 1997; Hazan \& Zeifman, 1994; Trinke \& Bartholomew, 1997). Among these behaviors, separation distress is considered a clear-cut marker of attachment in infant-parent relationships (Ainsworth et al., 1978; Sroufe \& Waters, 1977a) and in adult pair bonds (Hazan \& Zeifman, 1994; Hazan, Gur-Yaish, \& Campa, 2004).

Although human beings are born equipped to form attachment relationships, early interactions with caregivers shape the organization of attachment behaviors, thus leading to the development of an attachment style (Ainsworth et al., 1978). Ainsworth et al. (1978) identified three major infant attachment styles: secure, insecure-anxious, and insecure-avoidant. As a result of consistent and responsive caregiving, infants develop a secure attachment style. These infants tend to explore the environment as long as their caregiver is accessible but become distressed and cease exploring when separated from the caregiver. Upon reunion, they seek contact with and are comforted by the caregiver, and they eventually resume exploratory activities. When caregivers are inconsistent in their responses (i.e., sometimes available and soothing, sometimes neglecting and inaccessible), infants develop an insecure-anxious attachment style. Insecure-anxious infants usually fail to engage in exploration even in the presence of their caregiver. They become extremely distressed by separations from the caregiver. Upon reunion, they seek comfort from the caregiver but also angrily resist contact when it is established and thus are not fully soothed. Finally, when caregivers are consistently indifferent to or rejecting of infants' attachment behaviors (e.g., bids for contact), infants develop an insecure-avoidant attachment style. Insecureavoidant infants explore the environment in the presence of the caregiver, although in contrast to secure infants, they do not engage in affective sharing (e.g., smiling, showing the objects in the environment) with the caregiver. When separated from their caregiver, they surprisingly do not show overt signs of distress, and even more surprisingly they actively avoid contact with the caregiver upon reunion. However, other research indicates that their exploratory activity during separation is not associated with the deceleratory heart-rate response characterizing attentive exploration (Sroufe \& Waters, 1977b), which suggests distress at covert levels.

Similar individual differences have been observed in adults' romantic attachment patterns (Hazan \& Shaver, 1987). Initial studies (Hazan \& Shaver, 1987, 1990) identified three adult attachment styles - secure, anxious, and avoidant - that correspond to the three infant attachment styles. Current conceptualizations 
view individual differences in adult attachment not as a categorical style but as reflecting differences on two dimensions of insecurity: attachment-related anxiety and attachmentrelated avoidance (Brennan, Clark, \& Shaver, 1998; Fraley \& Waller, 1998). ${ }^{1}$ The first dimension, attachment-related anxiety, is characterized by a strong desire for closeness and protection in romantic relationships and intense worries about partner availability. The second dimension, attachment-related avoidance, is characterized by discomfort with closeness to and dependence on relationship partners and a strong preference for emotional distance.

Rather than reflecting the presence or absence of an attachment bond, these dimensions reflect differences in an individual's emotional and behavioral strategies to deal with the perceived inaccessibility of the attachment figure. For example, anxiously attached adults tend to perceive attachment figures as varying in their inaccessibility and responsiveness (sometimes being available and sometimes not). As a result, they try to capture the attention of and elicit care from attachment figures by intensifying expressions signaling a desire for proximity (referred to as a hyperactivation strategy; Main \& Hesse, 1990; Mikulincer \& Shaver, 2007). Indeed, research has found that anxiously attached individuals seek excessive reassurance from their romantic partner that they are loved (Shaver, Schachner, \& Mikulincer, 2005). Anxious individuals are also likely to behave negatively toward their partner after encountering stressful situations (Rholes, Simpson, \& Oriña, 1999). Maintaining these negative expressions, which signal unhappiness or distress, is part of anxious individuals' strategy to sustain proximity to an attachment figure that is perceived as inconsistently accessible (Cassidy, 1994), because showing signs of calmness and recovery increases the risk of losing contact with an inconsistently accessible partner. (Paradoxically, these negative behaviors may, in fact, increase a partner's dissatisfaction with

\footnotetext{
${ }^{1}$ In their original study, Ainsworth and colleagues (1978) also found that variation in infant attachment may be captured by two continuous dimensions. Subsequent taxometric analyses revealed that attachment styles are distributed continuously rather than categorically both in infancy and adulthood (Fraley \& Spieker, 2003; Fraley \& Waller, 1998). Readers who are interested in measurement of adult attachment styles may refer to Mikulincer and Shaver (2007) and Crowell, Fraley, and Shaver (2008).
}

the relationship and lead to eventual rejection in the long-term; Downey, Freitas, Michaelis, Khouri, 1998.) Avoidantly attached individuals, in contrast, rely on an opposite strategy. Because avoidantly attached adults perceive attachment figures as consistently inaccessible and unresponsive, they minimize proximity seeking and attempt to deal with stressors on their own (referred to as a deactivation strategy; Main \& Hesse, 1990; Mikulincer \& Shaver, 2007). Accordingly, research has found that avoidantly attached individuals are reluctant to overtly seek support from their partner in stressful situations (Collins \& B. C. Feeney, 2000). Regardless of differences in the organization of attachment behaviors, essentially, the function of all behavioral strategies is the same: They are involved in the regulation of felt security.

It is important to keep in mind that despite similarities between infant and adult attachment, adult pair bonds differ from infant-parent attachments in at least two important aspects (Hazan et al., 2004; Zeifman \& Hazan, 2008). First, in contrast to infant-caregiver attachments in which the parent meets the infant's attachment needs but not vice versa, reciprocal attachment and caregiving behaviors characterize pair bonds. Second, in addition to attachment and caregiving systems, pair bonds by definition involve the sexual mating system. In fact, early in a romantic relationship, sexual attraction is the primary motivation for seeking proximity to the partner (Hazan \& Zeifman, 1994). However, with time, the relative importance of sexual behaviors for maintaining the pair bond declines, whereas mutual attachment and caregiving come to play a more central role (Hazan \& Shaver, 1994). Indeed, research has shown that the frequency of sex in romantic relationships declines with time (e.g., Call, Sprecher, \& Schwartz, 1995), even though the relationships remain intact and the emotional bond strengthens.

In summary, attachment theory focuses on understanding the nature of the bond that characterizes our closest relationships across the life span. The theory has a normative component that describes the distinguishing features (proximity maintenance, safe haven, secure base, and separation distress) and function (regulation of felt security) of attachment relationships. The theory also has an individual difference component (i.e., attachment style) that explains different organizations of individuals' behaviors toward attachment figures. 


\section{ATTACHMENT IN MARRIAGE}

According to attachment theory, the pair bond, and hence its institutional form-marriage-is the prototypical attachment relationship in adulthood. Empirical evidence that marital relationships meet the definitional criteria of attachment relationships come from studies investigating to whom people direct attachment behaviors (i.e., proximity seeking, safe haven, separation distress, and secure base) in adulthood. Research recruiting participants from different ages (ranging from young to old adulthood) and marital status groups (single, dating, cohabiting, and married) has revealed that full-blown attachments, which involve all four classes of attachment behavior, are more likely to be observed with parents and romantic partners than with friends and adult children (Doherty \& Feeney, 2004; Fraley \& Davis, 1997; Hazan \& Zeifman, 1994; Trinke \& Bartholomew, 1997). Moreover, by adulthood, full-blown attachments with romantic partners (including spouses) outrank attachment to parents (Doherty \& J. A. Feeney, 2004; Hazan \& Zeifman, 1994), which suggests that spouses replace parents as primary attachment figures in adulthood.

Studies investigating marital separations provide further support that marital relations are attachment relationships. This work shows that reactions to separation from marital partners bear similarity to reactions to separation from early caregivers. Bowlby (1982) observed that when experiencing prolonged separation from their caregivers, infants show a predictable sequence of distress reactions. The initial reaction is to resist the separation by crying, searching, or calling out the caregiver to regain proximity. If unsuccessful, these attempts eventually yield to fatigue, depressed mood, sleep disturbances, and poor appetite. Finally, infants appear to recover and gradually build an interest in other activities and social relationships. Bowlby (1982) termed this sequence of reactions protest, despair, and detachment. Similarly, adults who have divorced from or lost their spouse as a result of death exhibit initial anxiety, panic, and anger followed by lethargy and depressed mood, and they eventually resume normal activities (Hazan \& Shaver, 1992; Parkes, 2006; Parkes \& Weiss, 1983; Weiss, 1975). Crucially, separation distress responses are likely to occur even when individuals are unhappy with their marriages (Weiss, 1975).
What are the implications of conceptualizing marriage as a pair bond? Given that a marriage, even one that is not very satisfying, constitutes an attachment relationship, attachment theory may contribute to the understanding of marital relationships in at least three ways. First, a basic function of marital relationships is to regulate spouses' felt security, which, in turn, provides psychological and physical health benefits. Second, with the felt security the attachment bond bestows comes increased exploration, which influences spouses' functioning outside the relationship, for example, their ability to meet personal goals. Third, attachment theory also allows for individual differences in a person's adult attachment style to influence attachmentrelated processes as well as marital adjustment.

We propose that the benefits conferred by the attachment bond in marriage (e.g., provision of felt security via the regulation of physiological and affective states and enhancement of exploratory activities) occur independent of the level of satisfaction experienced in the marriage and, at least to some extent, even in the absence of high marital satisfaction. We do not claim that these effects are realized in highly distressing or abusive marriages in which dysregulation may occur as a result of negative interactions. Rather, we argue that felt security, regulation of physiology and affect, and enhancement of exploration are typical provisions of marriage and do not necessarily require high levels of marital satisfaction.

To date, support for this proposition is limited to research focusing on moderately to highly satisfied individuals. Indeed, research has not yet specifically tested whether the effects of attachment relationship occur in dissatisfied couples. The restriction in range in levels of satisfaction couples have reported in the existing work limits the conclusions that can be drawn about the moderating role of satisfaction. Nonetheless, when reviewing relevant studies, we note whether the effects of attachment in marriage varied depending on the level of marital satisfaction.

We now elaborate on each of the contributions of attachment theory in turn.

\section{Spouses Provide and Regulate Each Other's Felt Security}

Given that marriage is an attachment relationship, one of its basic functions is to regulate spouses' felt security. Regulation of felt security 
in marital relationships may occur via two processes: stress buffering and synchrony (see also Hazan et al., 2004). The first aspect, stress buffering, refers to a process whereby spouses dampen each other's reactivity to stressful life events, thereby promoting stable levels of felt security even in the face of stressors. The second aspect, synchrony, refers to the coordination of spouses' psychological and physiological states over time. The primary factor contributing to both stress buffering and synchrony is the existence of the attachment relationship rather than its quality, although relationship quality may affect the magnitude of these effects.

Stress buffering. Stress buffering involves spouses' regulation of each other's reactivity to stressful events and is associated with important health implications. Stress buffering is thought to result from the neurobiological systems activated as a result of interactions with attachment figures and that are assumed to dampen the stress responses. Interactions with attachment figures, whether actual or symbolic, lead to increases in the activity of two neurotransmitter systems: oxytocin, which promotes feelings of trust, love, and affiliation, and endogenous opioids, such as endorphins, which decrease the experience of physical pain (Depue \& MorroneStrupinsky, 2005; Sbarra \& Hazan, 2008; Young $\&$ Wang, 2004). The same neurotransmitter systems also dampen stress-induced reactivity of the hypothalamic-pituitary-adrenocortical axis (HPA) and the autonomic nervous system (ANS) (Diamond, 2001; McCubbin, 1993; UvnäsMoberg, 1998), which are both implicated in various psychological and physical disorders, including generalized anxiety disorder, cardiovascular diseases, and immune deficits (Irwin, Hauger, \& Brown, 1992; Kamarck \& Jennings, 1991; McEwen \& Stellar, 1993; Thayer, Friedman, \& Borkovec, 1996). On the basis of these findings, researchers have argued that interactions with, or simply thinking about, attachment figures down-regulate HPA and ANS activity, which makes individuals less susceptible to the negative consequences of stressful events and provides protective health benefits (Diamond, 2001; Uvnäs-Moberg, 1998).

Evidence that spouses and partners dampen ANS reactivity comes from studies investigating married or cohabiting individuals' naturally occurring interactions (Gump, Polk, Kamarck, \& Shiffman, 2001; Holt-Lunstad, Uchino, Smith,
Olson-Cerny, \& Nealey-Moore, 2003). These studies found that interactions with spouses or partners were associated with significantly lower systolic and diastolic blood pressure (indicators of ANS activity) as compared to time spent alone or even interactions with other people. Furthermore, one of these studies (Gump et al., 2001) tested whether these effects were moderated by relationship quality and found that relationship quality was not significantly related to the buffering effect of partners on decrease in blood pressure, which suggests that the beneficial effects of spending time with one's partner occurs regardless of the level of satisfaction in the relationship.

Although these studies found that interactions with one's partner had a calming effect, at least physiologically, they are correlational in nature and do not establish that interactions with partners actually lead to decreases in ANS activation. For example, it is possible that individuals prefer to interact with partners when they are physiologically calmer or that they tend to interact with partners in situations that are less stress inducing. To test the directional hypothesis that interactions with partners leads to a reduction in reactivity, Grewen, Anderson, Girdler, and Light (2003) asked married or cohabiting participants either to engage in warm-contact activities with their partners (e.g., handholding while viewing a romantic video clip, hugging) or to spend time alone (e.g., resting quietly, standing alone) before a stressful task. Subsequently, participants were asked to deliver a public speech, a task that has been consistently shown to induce stress in laboratory settings (Dickerson \& Kemeny, 2004). Individuals in the warm-contact group demonstrated lower ANS reactivity, as indexed by significantly lower increases in systolic blood pressure, diastolic blood pressure, and heart rate during the speech task, than did those in the no contact group. Ditzen et al. (2007) observed buffering effects in HPA functioning as well, with warm partner contact leading to reduced cortisol response (an indicator of HPA activation) to a laboratory stressor. These findings suggest that contact with partners indeed down-regulates ANS and HPA reactivity in response to stressful events.

Moreover, research by Coan, Schaefer, and Davidson (2006) using functional magnetic resonance imaging (fMRI) has identified part of the neural circuitry underlying the stress-buffering effects of spouses. In this study, women in highly 
satisfying marriages were asked to hold their husband's hand, a stranger's hand, or no hand at all while anticipating a stressful event (a mild electric shock) in the scanner. The results revealed that holding the husband's hand, as compared to holding a stranger's hand or no hand at all, was associated with attenuation of neural activation in regions implicated in self-regulation of emotion and threat (caudate, right dorsolateral prefrontal cortex, and superior colliculus). In addition, in the spousal handholding condition, individuals who reported higher levels of marital quality showed greater neural attenuation in brain regions supporting the brain's stress response (right anterior insula, superior frontal gyrus, and hypothalamus). These findings corroborate the attachment perspective by showing that an available attachment figure provides a source of comfort in stressful situations, thereby reducing the need for regulation of emotion. Moreover, this work suggests that stress-buffering effects may be strongest among individuals in marital relationships characterized by high quality.

Recently, Master et al. (2009) extended these findings by showing that even the mental representation of the partner might suffice to buffer stress. They asked women in long-term romantic relationships to hold their partner's hand, to hold a stranger's hand, to view their partner's photograph, or to view a stranger's photograph while receiving thermal stimulation slightly higher than their pain thresholds. In line with Coan et al.'s (2006) findings, partner hand holding led to more reduced pain ratings than did stranger hand holding. Viewing the partner's photograph also led to lower pain ratings than viewing a stranger's photograph. These findings support the notion that even the mental representation of attachment figures may be sufficient to benefit from the security these individuals provide (Mikulincer \& Shaver, 2004; Uvnäs-Moberg, 1998).

Taken together, extant empirical evidence indicates that marital relationships, by virtue of being attachment relations, dampen responses to stressful events by way of reducing spouses' HPA and ANS reactivity. Indeed, it is striking that even a brief positive interaction with, or just seeing the photograph of, the partner (Coan et al., 2006; Grewen et al., 2003; Master et al., 2009) may be sufficient to buffer individuals from the effects of a stressful event. In daily life, repeated interactions of this sort may contribute to an enduring attenuation of stress reactivity in married individuals (Gump et al., 2001; HoltLunstad et al., 2003), which in turn protects them against psychological and physical health risks (see also Diamond, 2001).

Are stress-buffering effects magnified or dampened depending on marital quality? Tests of whether these effects are sensitive to marital quality have produced mixed results. On the one hand, Gump et al. (2001) failed to find a significant association between relationship quality and attenuation in blood pressure in interactions with the partner. On the other hand, Coan et al. (2006) found that marital quality was associated with additional benefits on neural systems regulating threat responses. A possible explanation of these findings is that stress buffering is a normative function of nondistressed marital relationships but may be augmented in highly satisfied marriages. (We return to this issue later in the Remaining Questions and Future Research Directions section.)

Synchrony. Synchrony is the coordination or comingling of spouses' psychological and physiological states over time. This coordination of psychological and physiological responses is likely to become internalized and contribute to an "emotional sense of security" (Feldman, 2007, p. 342), which we have been referring to as felt security throughout this article. Two differences between stress buffering and synchrony should be noted. First, stress buffering is primarily a unidirectional mechanism whereby one partner down-regulates the other partner's stress reactivity. Synchrony, however, is a bidirectional mechanism whereby both partners influence each other's physiological and psychological states. Second, stress buffering involves regulation of felt security before, during, or after stress exposure. Synchrony refers to a more general and enduring commingling of partners' psychological and physiological states.

The contribution of synchrony to felt security resembles the contribution of the external environment to the homeostasis, or stability, of psychophysiological systems. Psychophysiological systems in humans are open to regulation from external sources. Healthy functioning and effective self-regulation (e.g., planning, organizing, and pursuing goals; adjusting behaviors to environmental demands) depends on the intactness of these regulators. For example, external light-dark cycles regulate sleep-wake cycles and circadian rhythms, and changes in light-dark 
cycles cause the disruption of these rhythms. Researchers have argued that romantic partners serve a similar regulatory function (Ehlers, Frank, \& Kupfer, 1988; Hofer, 1994; Sbarra \& Hazan, 2008). As an attachment is formed between two partners, their psychological and physiological systems become synchronized to each other, and the relationship becomes a primary source of regulation for each individual. From an attachment perspective, synchrony between spouses helps each partner maintain their psychological and physiological homeostatic set-point from which they can function effectively (Sbarra \& Hazan, 2008).

Perhaps the strongest empirical support for synchrony comes from Feldman's (2007) studies of infant-mother attachment. In one study, Feldman found that when mothers effectively responded to their infant's cues during a play interaction, the mother's and the infant's heart rates became synchronized. That is, the infant's heart rhythms followed the deceleration or acceleration in the mother's heart rhythms. According to Feldman (2007), this coupling of physiological states with an attachment figure may lead individuals to internalize this experience as an emotional sense of security.

Studies similar to Feldman's are rare in the adulthood literature. One study found evidence for synchrony in romantic relationships at the level of subjective feelings (Butner, Diamond, $\&$ Hicks, 2007). Cohabiting couples (73\% were married) in highly satisfied relationships completed measures of positive and negative affect each day for three weeks. The researchers used these diary data to examine two forms of synchrony: affect covariation and affect coupling. Affect covariation refers to the tendency for partners' affective states to vary together. Affect coupling refers to the tendency of partners to influence each other's rates of change in affective cycles: Individuals oscillate between affective extremes in regular patterns that typically last for a few days, and coupling refers to the association between how quickly or slowly each partner is moving through their respective affective cycles. For instance, suppose that the husband's positive affect cycle normally follows a 5-day cycle (i.e., going from low to high to low positive affect in a 5 -day period). He starts a new job and his positive-affect cycle accelerates to a 4-day cycle. If there is coupling between the husband's and the wife's affect cycles, the acceleration in his cycle will accelerate her cycle as well. Butner et al. (2007) showed that partners' positive and negative affect covaried on a daily level. Moreover, the temporal coordination of partners' affective states was above and beyond any shared affect resulting from mutual positive and negative interactions. Strikingly, their positive affect cycles were coupled as well, indicating that the duration of one partner's positive affect cycle depended on that of the other partner. Another study (Saxbe \& Repetti, 2010) with married couples not only replicated the affect covariation findings for negative affect (though failed to do so for positive affect) but also extended them by providing evidence for covariation of daily cortisol levels between spouses. When one of the spouses' cortisol level was higher than usual, the other spouse's cortisol level also tended to be higher than usual. Further work is needed to understand the physiological and neural processes that are amenable to regulation by one's partner.

Work on synchrony in adult pair bonds is still in nascent stages. It is not surprising that the individual, relational, and contextual factors that may modulate synchrony have not been well studied. Saxbe and Repetti (2010) investigated, and found support for, the role of marital satisfaction on synchrony. Although cortisol levels of spouses covaried with the cortisol level of their partners at all levels of satisfaction represented in the sample, the covariation between cortisol levels was stronger for less satisfied (i.e., $1 S D$ below the mean) couples. Similarly, the covariation between spouses' negative mood was weaker for husbands who were highly satisfied (i.e., $1 S D$ above the mean; wives' level of satisfaction did not appreciably affect covariation). Two mechanisms may explain these findings. High marital satisfaction may prevent the negative influence of one spouse's HPA axis fluctuations on the other spouse's HPA functioning. Alternatively, couples who are less influenced by elevations in each other's cortisol levels become more satisfied with their relationships. Future work is needed to understand the causal relationships between marital satisfaction and synchrony in couples' psychological and physiological states.

Given that synchrony appears to be a defining feature of attachment relationships, it is not surprising that separation from or loss of a spouse disrupts synchrony. Disruption of synchrony has important implications for understanding the physical and psychological 
reactions to separations, even when relationships are not highly satisfying. When spouses separate, they lose one of the factors regulating their physiological and psychological systems, and as a result, the systems become dysregulated. Indeed, in a review of retrospective studies on wartime and job-related marital separations, Vormbrock (1993) found that separation from the spouse induced anxiety, depression, sleep disturbances, poor appetite, and weight change. Moreover, simply replacing the spousal tie with another social tie did not assuage these disturbances. A limitation of these studies, however, was their retrospective design. Diamond, Hicks, and Otter-Henderson (2008) addressed this limitation by collecting daily measures of affect, behavior, and physiology (HPA activation) before, during, and after a brief separation lasting for 4-7 days. They found that couples experienced a decrease in positive mood and an increase in sleeping problems as a result of separation. Moreover, reunion counteracted these changes; for example, positive affect increased and sleep patterns returned to normal. Most important, marital satisfaction did not significantly amplify or dampen the separation or reunion effects.

As the present section shows, attachment theory makes its first contribution to marital research by defining a normative function of marital relationships (i.e., regulation of felt security). Regulation of felt security in a marital relationship depends on two processes: stress buffering and synchrony. Evidence suggests that both processes operate in marital relationships. In fact, researchers have argued that these dynamics may create a "psychological tether" (Hazan \& Zeifman, 1994) that keeps spouses together, even when they are not happy with being together anymore. Indeed, this might be one reason why some people return to relationships despite intentions to separate (Kitson \& Raschke, 1981).

\section{Spouses Facilitate Exploration}

Whether in infancy or in adulthood, the attachment bond provides individuals with a secure base from which to explore the world. In infancy, proximity to the parent is an important determinant of exploration. In adulthood, actual or imagined proximity to marital partners provides the primary secure base from which individuals explore the world. As Bowlby (1988) aptly put it, we are "happiest when life is organized around a series of excursions, long or short, from the secure base provided by our attachment figures" (p. 62). In adulthood, such exploratory excursions may take the form of goal pursuits, such as developing a new hobby, switching jobs, or losing weight, and the ability to achieve them. Thus, the second contribution of attachment theory to marital research is that it makes predictions about partners' functioning outside the relationship, especially with regard to their ability to engage in exploratory behavior and pursue a variety of personal goals.

B. C. Feeney (2004) investigated the secure base component of adult attachments with a sample of individuals in committed relationships $(70 \%$ were cohabiting or married). Findings revealed that participants' perception of their partner as responsive to their needs was positively associated with their self-reported willingness to approach exploratory opportunities (e.g., "I am usually willing to accept challenges and try new things"') and perceived likelihood of achieving their personal goals. Furthermore, individuals who discussed their personal goals with a supportive partner showed a greater increase in their perceived likelihood of achieving their personal goals than did individuals who discussed their personal goals with a less supportive or unsupportive partner. Moreover, B. C. Feeney and Thrush (2010) observed that having an available, encouraging, and nonintrusive spouse was associated with higher persistence in and enthusiasm about a laboratory exploration activity (a puzzle-solving task).

A limitation of this work is that all measures are assessed concurrently. However, these findings have also been supported in a longitudinal study (B. C. Feeney, 2007) with a sample of married couples. Participants who had spouses who were more sensitive and responsive toward them were more likely to report achieving a personal goal at the end of six months than were participants with less sensitive and responsive spouses. Moreover, spouses' sensitivity and responsiveness toward participants' needs were positively associated with participants' selfreported willingness to engage in exploratory activities six months later, even after controlling for initial willingness to engage in exploration.

An important exploratory goal in adulthood is to achieve one's ideal self. Rusbult and colleagues (Drigotas, Rusbult, Wieselquist, and Whitton, 1999; Kumashiro, Rusbult, Wolf, and 
Estrada, 2006) proposed that one's romantic partner "sculpts" one to be her ideal self through a process called the Michelangelo phenomenon. According to this model, the partner's perceptual and behavioral affirmation facilitates one's movement toward the ideal self. Perceptual affirmation refers to the degree to which one's partner "believes" that one can acquire the ideal self. Behavioral affirmation refers to the degree to which one's partner "behaves" in a way that elicits ideal-congruent qualities in one's self. In several studies, the authors demonstrated that one partner's perceptual affirmation led to higher behavioral affirmation, which, in turn, increased the other partner's movement toward the ideal self (Drigotas et al., 1999; Kumashiro et al., 2006). Moreover, in longitudinal studies, partner behavioral affirmation predicted movement toward the ideal self at a later time in both dating and married couples (Drigotas et al., 1999; Kumashiro et al., 2006).

Given that simply thinking about one's spouse or romantic partner confers stress buffering effects (Master et al., 2009), Selcuk and Hazan (2010) investigated whether simply activating the mental representation of one's romantic partner is sufficient to increase individuals' motivation to engage in exploration. That is, although past work (Drigotas et al., 1999; B. C. Feeney, 2004, 2007; Kumashiro et al., 2006) has shown that explicit, overt supportive behaviors (e.g., being sensitive to needs, supporting goal pursuit, behavioral affirmation) influence exploration-related attitudes and behaviors, in these studies, participants were asked to just think about either their romantic partner or various social or nonsocial topics before completing measures of curiosity and exploratory behavior. Findings revealed that thinking about the partner significantly increased curiosity and exploratory behaviors as compared with thinking about control topics. Moreover, these effects were not significantly affected by relationship satisfaction. Although this work has not been conducted with a sample of married individuals (yet), the initial findings indicate that even the symbolic presence of attachment figures may increase one's curiosity and exploratory tendency.

Overall, studies examining the secure-base hypothesis in adulthood emphasize another route for spousal attachments to improve wellbeing, likely independent of the contribution of relationship satisfaction. By both being available when needed and actively bringing out the qualities that individuals are aspiring to achieve, attachment figures provide a base from which to pursue exploratory opportunities and work toward personally meaningful goals.

\section{Quality of Attachment Shapes Marital Functioning}

Do individual differences in adult attachment impact normative attachment dynamics such as stress buffering, synchrony, and facilitation of exploration? In the following section, we review the literature on the role of individual differences in attachment style and how it may offer a third contribution to marital research.

Adult attachment styles are measured along two orthogonal dimensions: The first dimension, attachment-related anxiety, is characterized by a strong desire for closeness and protection in romantic relationships and intense worries about partner availability. The second dimension, attachment-related avoidance, is characterized by discomfort with closeness to and dependence on relationship partners and a strong preference for emotional distance. Individuals who score low on these two dimensions are assumed to have a secure attachment style.

Studies examining the role of attachment style in marital functioning generally fall into one of two categories: (a) studies examining whether attachment style predicts marital adjustment, including satisfaction, stability, and conflict management and (b) studies examining whether attachment style moderates the normative attachment processes of obtaining felt security and increasing exploration. The vast majority of existing studies fall under the first category (for a comprehensive review of these studies, see Mikulincer \& Shaver, 2007). Numerous crosssectional and longitudinal studies have consistently documented that both attachment-related anxiety and attachment-related avoidance are negatively associated with marital satisfaction (e.g., Berant, Mikulincer, \& Florian, 2003; Carnelley, Pietromonaco, \& Jaffe, 1996; Cobb, Davila, \& Bradbury, 2001; Davila \& Bradbury, 2001; Davila, Bradbury, \& Fincham, 1998; Rholes, Simpson, \& Friedman, 2006). Studies examining relationship stability have found that attachment-related avoidance is positively related to divorce and multiple marriages (e.g., Ceglian \& Gardner, 1999; Hill, Young, \& Nord, 1994), whereas attachment-related anxiety is positively associated with staying in an unhappy 
marriage (Davila \& Bradbury, 2001). Studies investigating conflict management have found that both attachment-related anxiety and avoidance are positively associated with destructive and coercive conflict management behaviors and negatively associated with constructive and compromising behaviors (e.g., Carnelley, Pietromonaco, \& Jaffe, 1994; J. A. Feeney, 1994; Marchand, 2004). One study observed that spouses' commitment in marriage buffered the negative effects of attachment anxiety on conflict management (Tran \& Simpson, 2009). Finally, studies investigating violence in couples have found that attachment insecurity, especially attachment-related anxiety, is associated with the occurrence and severity of violence in marriage (e.g., Dutton, Saunders, Starzomski, \& Bartholomew, 1994; Holtzworth-Munroe, Stuart, \& Hutchinson, 1997; Rankin, Saunders, \& Williams, 2000).

Studies in the second category - those examining the effect of attachment style on stress buffering, synchrony, and exploration - are relatively few in number. Although additional work is needed, the emerging picture is one in which individuals who are high on avoidance show less use of their spouse for regulatory functions (i.e., synchrony and stress buffering). Regarding synchrony between partners, Butner et al. (2007) found that avoidant individuals were less likely to be influenced by changes in their partner's positive affect cycles than were nonavoidant individuals. Using fMRI, Coan (2008) found that, during husband hand holding, secure women showed less activation in areas implicated in affect-related arousal, whereas avoidant women showed increased activation in areas implicated in negative affect regulation (see Coan, 2008). This finding suggests that avoidant individuals might be less likely to benefit from stress buffering than nonavoidant individuals.

Whereas avoidant individuals fail to optimally benefit from regulatory functions of attachment bonds, anxious individuals show more severe responses to the bond's disruption (e.g., separation). For example, Diamond et al. (2008) found that short-term separations from the partner were associated with heightened HPA activation for only anxious individuals. Upon reunion with the partner, HPA activity returned to preseparation levels for these individuals. Thus, although in general individuals may experience less positive affect and greater sleeping problems as a normative consequence of short-term separation from the partner, attachment-related anxiety may exacerbate separation distress. Indeed, one study found that even imagining a separation from the romantic partner increased the mental accessibility of death-related thoughts in anxiously attached individuals (Mikulincer, Florian, Birnbaum, \& Malishkevich, 2002).

Despite these findings, in studies investigating the secure base component of attachment relationships that is critical for promoting exploration, neither B. C. Feeney (2007) nor Selcuk and Hazan (2010) found that attachment style appreciably changed the effect of the partner on exploration and goal pursuit. However, B. C. Feeney and Thrush (2010) observed that anxious and avoidant individuals' spouses were less available when these individuals were performing an exploratory task (a puzzle-solving task) in the laboratory. Moreover, avoidant individuals received less encouragement from their spouses during exploration. Although more work is needed, these findings suggest that attachment style may moderate the effect of spouses on exploration by way of perceptions, or actual receipt, of spousal support. Moreover, other studies investigating the link between a person's attachment style and chronic, dispositional levels of exploratory behaviors found that both attachment-related anxiety and avoidance were related to lower exploration (Elliot \& Reis, 2003; Green \& Campbell, 2000; Hazan \& Shaver, 1990).

In summary, although attachment style is associated with and predicts several marital outcomes (e.g., satisfaction, stability, conflict management), there have been relatively few studies examining whether attachment style moderates partners' stress-buffering effects, synchrony in romantic relationships, and partners' facilitation of exploratory activities. These studies found that attachment style was associated with synchrony and stress buffering but did not have a significant effect on partners' facilitation of exploration. As the number of such studies increases, we will have a better idea of the interplay between these processes and individual difference factors.

\section{REMAINING QUESTIONS AND FUTURE RESEARCH DIRECTIONS}

Despite what is currently known about attachment processes in marriage, both at the 
normative level and the individual difference level, the field is ripe for further inquiry. We briefly outline future directions for research on marital relationships that incorporates an attachment perspective.

\section{Role of Marital Satisfaction}

Current evidence suggests that marital relationships serve attachment functions, at least to some extent, independent of satisfaction. In two studies, marital satisfaction was found to affect stress buffering and synchrony effects, with spouses in highly satisfying marriages experiencing greater stress buffering (Coan et al., 2006) and less covariation in cortisol levels (Saxbe \& Repetti, 2010). Differences in marital satisfaction did not completely account for stress buffering and synchrony effects in either of these studies but modulated the effects' strength. Other studies (Diamond et al., 2008; Gump et al., 2001) failed to find any appreciable effect of marital satisfaction on synchrony and stress buffering. Taken together, these findings suggest that stress buffering, synchrony, and enhancement of exploration may be more or less observed within the normal range of marital satisfaction. However, this conclusion is only tentative, as none of the existing studies specifically recruited couples who were very low in satisfaction or couples who were highly distressed and experiencing a high frequency and escalation of negative interactions (Fincham \& Beach, 1999). In distressed relationships, the relationship itself may be a source of stress instead of security. Indeed, some researchers have argued that highly distressed marriages might be as detrimental to physical and mental health as social isolation (Robles \& Kiecolt-Glaser, 2003). Future studies recruiting highly satisfied, highly dissatisfied, and clinically distressed couples may help elucidate the role of relationship satisfaction in attachment processes.

\section{Unpacking Synchrony}

To date, studies provide convincing, albeit initial, evidence for the occurrence of synchrony in affective states between spouses (e.g., Butner et al., 2007; Diamond et al, 2008). However, numerous researchers have proposed that synchrony also instantiates at the level of physiology (Hofer, 1994; Polan \& Hofer, 2008; Sbarra \& Hazan, 2008). Although initial findings provided by Saxbe and Repetti (2010) are encouraging, we need further experimental tests of synchrony in spouses' physiological states. For instance, future studies may assess experimentally induced changes in ANS and HPA functioning and test the degree to which one individual's current physiological state depends on the spouse's prior physiological state as well as on his or her own prior physiological state.

\section{Attachment-Exploration Link}

If the maintenance of an attachment bond promotes exploration as shown in several studies, what are the costs of the bond's disruption to exploration? Attachment theory predicts that separation from the spouse reduces felt security and, as a result, interferes with exploratory behaviors. Recently, Cavallo, Fitzsimons, and Holmes (2010) reported that dating individuals who wrote about a threat to their relationship showed higher mental accessibility to avoidance-related words (e.g., safety, prevent) and lower accessibility to approach-related words (e.g., eager, progress). Given that highapproach and low-avoidance orientations might indicate exploratory tendencies, this study provides indirect support for the prediction of attachment theory. To our best knowledge, no study so far has conducted a direct test of this prediction by examining changes in exploratory behaviors following separations from or loss of a spouse. Such studies will be invaluable additions to the body of knowledge about the interplay between attachment and exploration in adulthood.

\section{Marital Therapy}

Research on marital satisfaction has provided marital therapists with invaluable tools for developing effective interventions that aim to improve marital functioning. Attachment theory offers a complementary tool by suggesting that one way to assist couples in strained marriages is to help them restore the security regulating function of the relationship. Recent research on the effectiveness of the emotionally focused model of couple therapy (EFT) (Johnson, 2004), a model of intervention heavily relying on attachment principles, provides an excellent case in point. Research shows that a strong predictor of divorce is the demand-withdraw 
pattern, where one spouse angrily accuses the other spouse and makes emotional demands and the other spouse defensively withdraws from the communication (Gottman, 1994). A therapist using EFT interprets angry accusations and demands as reactions to perceptions of the spouse as unavailable and unresponsive to meet attachment needs (i.e., support, comfort, and protection). The therapist first shows spouses that maintaining the demand-withdraw pattern fuels the demanding spouse's attachment-related fears (i.e., losing the attachment figure) and then guides spouses to deescalate the pattern. Next, the therapist helps the withdrawing spouse to articulate the attachment figure role and encourages the couple to openly ask each other for comfort and support. Moreover, by acknowledging individual differences, the therapist may tailor the therapy according to the attachment style of each spouse. For instance, the therapist may help an anxiously attached spouse to communicate attachment needs without destructive expressions of anger and hostility or may encourage an avoidant spouse to reduce the barriers in front of emotional engagement. Studies have shown that the EFT is associated with positive treatment outcomes, as indicated by alleviation or elimination of marital distress symptoms (Johnson, Hunsley, Greenberg, \& Schindler, 1999). Moreover, there is evidence for the stability of treatment outcomes over time (Cloutier, Manion, Gordon Walker, \& Johnson, 2002). Future research should examine whether attachment-based interventions also enhance couples' coping with stressful life events (e.g., transition to parenthood, financial difficulties).

Moreover, research and theory on attachment also suggests the possibility of new marital interventions that involve promoting excitement and arousal in marriage. As discussed, marital relationships provide feelings of security. However, most people, at least in Western societies, want their marriages to be not only sources of safety and security but also sources of excitement and arousal. Does attachment theory speak to this phenomenon? Extrapolating the theory's premise about the interplay between attachment and exploration, spouses' joint participation in exploratory activities may be one way to sustain feelings of excitement and arousal in marriage. Just like individual exploration from the secure base is rewarding for individual wellbeing, shared exploration with the secure base may be rewarding for the relationship. In parallel to this idea, A. Aron and E. Aron's (2006) selfexpansion model suggests that joint participation in new activities promote the maintenance of romantic love. Both correlational and experimental studies with married couples (A. Aron, Norman, E. Aron, McKenna, \& Heyman, 2000) found that shared participation in novel and challenging activities led to higher satisfaction and excitement with the relationship. Future research should systematically integrate attachment theory and the self-expansion model to test the ways in which shared exploration with the spouse increases feelings of excitement and arousal in marriage.

\section{Attachment Style: General or Relationship Specific?}

Is attachment style a general, traitlike individualdifference variable or a relationship-specific variable? For example, is it a characteristic that is relatively consistent across relationships and one that guides feelings, thoughts, and behaviors irrespective of the characteristics of the relationship partners? Or is it a characteristic that varies depending on the nature of the specific relationship and the characteristics of the relationship partner? This issue is of importance because it speaks to whether, for example, an individual with an avoidant attachment style is able, according to the theory, to form and maintain a secure marital relationship.

Research using primarily unmarried, collegeaged samples has found that individuals have a dominant style of relating to others in their close relationships, which could be referred to as their chronic attachment style (e.g., Baldwin, Keelan, Fehr, Enns, \& Koh-Rangarajoo, 1996; Cozzarelli, Hoekstra \& Bylsma, 2000). Nonetheless, they do not simply have a single style. Indeed, researchers have observed low to moderate variation in attachment style over time, ranging from 1 week (Baldwin \& Fehr, 1995) to 27 years (Klohnen \& Bera, 1998; for a meta-analysis of available studies, see Fraley \& Brumbaugh, 2004) and across different relationships (Baldwin et al., 1996; Cozzarelli et al., 2000). To account for this variability, it has been suggested that individuals have available to them various mental models of relationships, acquired either directly via their own interpersonal experiences or indirectly via observation (Baldwin, et al. 1996), and the mental model activated at a given time will influence the thoughts and 
feelings accessible, as well as individual's subjective experience and behaviors.

These findings have important implications for marital research. They suggest that one's attachment style toward a spouse is affected not only by the individual's general attachment style (i.e., chronic ways of feeling, thinking, and behaving with attachment figures) but also by current experiences with his or her partner. Adults who have a secure attachment style may become insecurely attached to their spouse if they face rejection, disapproval, or negligence from the spouse. Similarly, an adult who has an insecure attachment style may become securely attached to a spouse who is consistently available, responsive, and supportive. Thus, according to attachment theory, one's chronic attachment style neither guarantees happiness in marriage nor destines oneself to a malfunctioning relationship. Rather, the nature of the current relationship is an important determinant of one's organization of attachment behaviors in that relationship. In line with this argument, one study found that negative interpersonal experiences such as disapproval of or separation from a partner on a given day predicts increases in attachment anxiety and avoidance on that day, even after controlling the previous-day levels of anxiety and avoidance (Davila \& Sargent, 2003). Another study showed that transition to marriage moves spouses toward secure attachment if they have favorable appraisals of their marriage (Davila, Karney, \& Bradbury, 1999).

In a separate line of work attempting to understand the relationship-specific role of attachment style, researchers defined secure or insecure couples on the basis of pairings of the attachment styles of each spouse. Some of the studies found that secure couples (couples in which both partners were securely attached) were more satisfied with their marriages than insecure couples (i.e., couples in which both partners were insecurely attached) (W. H. Berman, Marcus, \& E. R. Berman, 1994; Senchak \& Leonard, 1992). Another study reported that the difference in marital satisfaction occurs only when spouses in insecure couples score high on both attachment anxiety and avoidance but not when one of the spouses score low on one of the dimensions (Ben-Ari \& Lavee, 2005). Still another study found no differences in marital satisfaction between couple types (Volling, Notaro, \& Larsen, 1998). Given the small number of studies and discrepant findings, further research is needed to understand the role of individual versus relationship-level effects on attachment processes and marital outcomes.

\section{Culture, Context, and Diversity}

Another important future research avenue concerns the effects of contextual (e.g., socioeconomic status, culture) and individual (e.g., gender, race, sexual orientation) factors on attachment processes in marital or other longterm romantic relationships. Here, we review the literature on the role of culture, ethnicity, gender, and sexual orientation in attachment processes and identify questions for future research.

Culture. According to attachment theory, humans as well as nonhuman primates and other species have evolved to form attachments with conspecifics. Indeed, researchers observed proximity seeking, separation distress, and stress buffering in primate mother-infant dyads and some primate pair bonds (e.g., titi monkeys; Mason \& Mendoza, 1998). This basic need to form attachments is presumed to supersede cultural and contextual differences. Thus, attachment theory predicts that all individuals - when given the opportunity - become attached to one or more specific figures. This prediction is referred to as the universality hypothesis, which has received considerable support in studies conducted with caregivers and infants in different parts of the world from North America and West Europe to East Asia and Africa (van IJzendoorn \& Sagi-Schwartz, 2008). Specifically, regardless of childrearing arrangements and family constellations, infants direct attachment behaviors - proximity seeking, safe haven, separation distress, and secure base-toward their caregivers (for a review, see van IJzendoorn \& Sagi-Schwartz, 2008).

The universality hypothesis has not been directly tested in adulthood. Animal models (Mason \& Mendoza, 1998) imply that human attachments to mates is a universal phenomenon and human adults' predilection to form selective and enduring relationships to mates is observed across cultures (Goodwin, 1999; Rosenblatt \& Anderson, 1981). At the same time, however, it is important to note that in some cultures, such as the United States, approximately $50 \%$ of the population 15 years and older is married, which means that a substantial proportion of individuals have never been married $(30.8 \%)$, are divorced (10.6\%), or widowed (6.3\%; U.S. 
Census Bureau, 2000). Although these statistics do not speak to the proportion of individuals who are unmarried but in a pair bond, there is still a substantial proportion who are not in a pair bond. This raises questions regarding the universality hypothesis in adulthood and regarding how single, divorced, or widowed individuals meet their attachment needs outside of a pair bond.

Moreover, cultures vary widely in the precise nature of attachment ties in adulthood, for example, from monogamy to polygyny or polyandry (Goodwin, 1999). Do these cultural variations influence attachment processes in adulthood (e.g., use of partners as secure bases)? Scant cross-cultural studies exist investigating these types of questions. However, studies investigating marriage norms in different cultures (i.e., self-selected marriages vs. arranged marriages) have shown that attachment behaviors between spouses occur in both self-selected and arranged marriages (Blood, 1967; Wynne, 1988). These findings constitute some evidence for the universality hypothesis in adulthood. Nevertheless, to better understand the role of culture on the tendency to form attachments in adulthood, future studies explicitly comparing adult attachment formation and maintenance in different cultures are needed.

The argument that forming attachments with caregivers in infancy and with romantic partners in adulthood is universal does not mean that the expression of attachment behaviors, as reflected by a person's attachment style, will be the same across all contexts. Belsky and colleagues (Belsky, 2010; Belsky, Steinberg, \& Draper, 1991) argued that contexts characterized by high levels of stress and economic hardship, through their effects on early caregiving behaviors, increase the likelihood of developing an insecure attachment style. Schmitt et al. (2004) conducted one of the most comprehensive studies examining this idea in an adult sample of 17,804 individuals from 62 cultural regions. Using the United Nation's Human Development Index (HDI, an indicator of a country's achievement in basic human capabilities, including health, longevity, education, and standard of living) and the national gross domestic product (GDP) as indicators of stress and hardship, they found, as predicted by Belsky and colleagues, that individuals from regions with lower levels of HDI and GDP were more likely to have greater attachment-related anxiety and avoidance in adulthood.
In summary, current evidence suggests that individuals' tendency to form attachments with parents in infancy is universal. There is also evidence that adults form pair bonds across cultures, although there is more variability in the organization of pair bonds across cultures. The fact that there is less evidence to support the universality hypothesis in adulthood (than in infancy) highlights some of the differences between infant and adult attachment we discussed earlier. In infancy, attachment to the primary caregiver is necessary for survival. Infants who do not remain close to attachment figures are more likely to suffer injury and illness, because they are unable to care for themselves. In adulthood, attachment to partners is associated with physical and psychological health benefits. Nonetheless, in adulthood pair bonds are not necessarily a requirement for survival, as adults are better equipped to take care of themselves, especially in a community of family and friends. Finally, the expression of attachment behaviors (e.g., distribution of attachment styles) across contexts differs with greater levels of stress and economic hardship leading to higher percentages of insecure attachment styles. More research investigating the universality hypothesis in adulthood and whether and how cultural norms influence attachment process are needed.

Ethnicity. Unfortunately, most of the research on adult attachment has relied on individuals of Caucasian and Western descent. Indeed, most studies described in our review have recruited Caucasian participants. To our knowledge, there are only two studies that recruited and directly compared Caucasian and African American participants. In the Gump et al. (2001) study, interactions with spouses or partners were associated with lower ANS activity for both Caucasian $(n=60)$ and African American participants $(n=60)$. Similarly, in the Grewen et al. (2003) study, both Caucasian $(n=117)$ and African American $(n=66)$ participants experienced attenuated stress reactivity during the public speech task following warm contact with their spouses or partners, although the benefits were significantly greater for African American participants. The absence of research using samples of diverse ethnic and racial compositions means that, as a field, we know very little about the role of race and ethnicity in adult attachment, and efforts to recruit individuals from diverse ethnic and racial backgrounds are needed. 
Gender. Regarding gender differences, studies examining synchrony, stress buffering, and exploration found comparable effects for both women and men, with the exception of two studies (Diamond et al., 2008; Saxbe \& Repetti, 2010). Diamond et al. (2008) found an increase in negative affect following a temporary separation for only homebound female partners but not for homebound male partners. Note that Diamond et al. (2008) did not find any significant gender differences in terms of change in positive affect, sleeping problems, and cortisol levels in the same sample. Saxbe and Repetti (2010) observed that marital satisfaction weakened covariation of cortisol levels for both husbands and wives. However, it weakened the covariation of negative affect for only husbands but not for wives. No notable gender differences were observed in studies examining the effect of spouses' attachment styles on marital outcomes such as satisfaction, stability, or conflict management (see also Mikulincer \& Shaver, 2007).

Although there have only been a few studies documenting gender differences in synchrony, stress buffering, and exploration, other research suggests that the ease with which the attachment system is activated and deactivated, the types of threats that may activate threat responses and therefore the attachment system, and the expression of attachment behaviors may differ for men and women. For example, compared to men, women appear to be more sensitive to interpersonal threat (Ayduk, Downey, \& Kim, 2001), are better able to detect nonverbal and emotional expressions (McClure, 2000; Neff \& Karney, 2005), and express more emotions, such as crying (Becht \& Vingerhoets, 2002). To the extent that they serve to activate and deactivate the attachment system, differences in the perception, experience, expression, and regulation of emotion (for a review, see Vigil, 2009) may play a role in the formation and maintenance of attachment relationships as well as the expression of attachment behaviors. Moreover, provocative work by Diamond (2003) suggests that the link between sexual desire and romantic love, both of which are key components of the pair bond in adulthood, may differ for men and women.

An important question with respect to the issue of gender is whether there is a gender difference in attachment styles. Studies investigating this question have produced mixed results. Whereas some studies have found that men tend to rate themselves as more avoidant and less anxious than women (e.g., Brassard, Shaver, \& Lussier, 2007; Brennan et al., 1998; Scharfe \& Bartholomew, 1994), other studies found no gender differences (e.g., Hazan \& Shaver, 1987; Gentzler \& Kerns, 2004; Jang, Smith, \& Levine, 2002). In their study conducted in 62 cultural regions, Schmitt et al. (2003) also examined gender differences in adult attachment styles. Their findings revealed that men scored higher on attachment-related avoidance than women did. The effect size of the gender difference, however, significantly varied as a function of culture. For example, Hawaii, Northern Ireland, and Latvia showed moderate effect sizes (Cohen's $d \mathrm{~s}>.40$ ), and Canada (English); northeastern, western, and southern United States; Peru; Argentina; and Turkey showed small to moderate effect sizes (Cohen's $d$ s ranged from .20 to .39). However, some cultures, such as Canada (French), Finland, and Lebanon, showed trivial effects (Cohen's $d \mathrm{~s}<.10$ ), whereas others, such as Tanzania, showed nonsignificant reversals (women being more avoidant than men). The authors concluded that greater male avoidance is " near universal," though variable across cultures (p. 322).

In summary, our qualitative review of the literature did not provide a conclusive picture of gender differences in adult attachment. Unfortunately, to the best of our knowledge, there has been no meta-analysis combining the findings of existing studies examining whether gender differences exist in adult attachment style.

Sexual orientation. With respect to sexual orientation, there is no theoretical reason to assume that attachment-related processes would differ across heterosexual and same-sex couples. Similar to heterosexual individuals, gay or lesbian individuals direct attachment behaviors toward their romantic partners (Diamond \& Dubé, 2002; Mackey, Diemer, \& O'Brien, 2004). Moreover, individual differences in attachment style affect relationship functioning in the same way in both heterosexual and same-sex couples (Mohr, 2008). However, there are still unanswered questions. For instance, does the experience of stressors, such as perceived discrimination, disapproval of others, and so on, affect security regulation in same-sex couples? Future work is needed to gain a better understanding of attachment dynamics in samesex romantic relationships. 


\section{CONCLUDING REMARKS}

Marital research has shed light into the factors that contribute to marital satisfaction and stability. In this article, we aimed to complement this ongoing effort with an attachment theory perspective. We proposed and provided evidence that marital relationships, even in the absence of high satisfaction, serve attachment functions. Accordingly, we argued that a basic function of marriage is to regulate spouses' felt security and that the maintenance of the attachment bond and associated felt security enhances spouses' physical and psychological functioning.

Numerous studies by different research teams in different fields have provided strong support for this argument. Specifically, studies have shown that spouses buffer each other's stress reactivity, which is associated with protective health benefits. Moreover, spouses' psychological and physiological systems are synchronized and marital separation results in dysregulation of these systems. In addition, maintaining an attachment bond with a security providing spouse promotes functioning outside the relationship by facilitating exploration. Finally, studies reported that individual differences in attachment style shape marital functioning via directly affecting marital adjustment or via moderating synchrony and stress buffering.

As the present review shows, much can be gained by an integration of the attachment and marital literatures. Given that the focus of the current volume is marital relationships, we sought to show how attachment theory contributes to marital research. Surely, there is much to be gained by applying findings and theory from marital research to understanding attachment dynamics. We hope this article promotes the necessary dialogue between attachment and marital researchers to achieve a better understanding of the nature of marital relationships.

\section{REFERENCES}

Ainsworth, M. D. S. (1991). Attachment and other affectional bonds across the life cycle. In C. M. Parkes, J. Stevenson-Hinde, \& P. Marris (Eds.), Attachment across the life cycle (pp. 33-51). New York: Routledge.

Ainsworth, M. D. S., Blehar, M. C., Waters, E., \& Wall, S. (1978). Patterns of attachment: Assessed in the strange situation and at home. Hillsdale, NJ: Erlbaum.

Aron, A., \& Aron, E. N. (2006). Romantic relationships from the perspectives of the self-expansion model and attachment theory. In M. Mikulincer \& G. S. Goodman (Eds.), Dynamics of romantic love: Attachment, caregiving, and sex (pp. 359-382). New York: Guilford Press.

Aron, A., Norman, C. C., Aron, E. N., McKenna, C., \& Heyman, R. (2000). Couples' shared participation in novel and arousing activities and experienced relationship quality. Journal of Personality and Social Psychology, 78, 273-283.

Ayduk, O., Downey, G., \& Kim, M. (2001). Rejection sensitivity and depressive symptoms in women. Personality and Social Psychology Bulletin, 27, $868-877$.

Baldwin, M. W., \& Fehr, B. (1995). On the instability of attachment style ratings. Personal Relationships, 2, 247-261.

Baldwin, M. W., Keelan, J. P. R., Fehr, B., Enns, V., \& Koh Rangarajoo, E. (1996). Social-cognitive conceptualization of attachment working models: Availability and accessibility effects. Journal of Personality and Social Psychology, 71, 94-109.

Becht, M. C., \& Vingerhoets, A. J. J. M. (2002). Crying and mood change: A cross cultural study. Cognition and Emotion, 16, 87-101.

Belsky, J. (2010). Childhood experience and the development of reproductive strategies. Psicothema, 22, 28-34.

Belsky, J., Steinberg, L., \& Draper, P. (1991). Childhood experience, interpersonal development and reproductive strategy. Child Development, 62, $647-670$.

Ben-Ari, A., \& Lavee, Y. (2005). Dyadic characteristics of individual attributes: Attachment, neuroticism, and their relation to marital quality and closeness. American Journal of Orthopsychiatry, $75,621-631$.

Berant, E., Mikulincer, M., \& Florian, V. (2003). Marital satisfaction among mothers of infants with congenital heart disease: The contribution of illness severity, attachment style, and the coping process. Anxiety, Stress, and Coping, 16, 397-415.

Berman, W. H., Marcus, L., \& Berman, E. R. (1994). Attachment in marital relations. In M. B. Sperling \& W. H. Berman (Eds.), Attachment in adults: Clinical and developmental perspectives (pp. 204-231). New York: Guilford Press.

Blood, R. O., Jr. (1967). Love match and arranged marriage: A Tokyo-Detroit comparison. New York: Free Press.

Bowlby, J. (1973). Attachment and loss: Vol. 2. Separation: Anxiety and anger. New York: Basic Books.

Bowlby, J. (1979). The making and breaking of affectional bonds. London: Tavistock.

Bowlby, J. (1980). Attachment and loss: Vol. 3. Sadness and depression. New York: Basic Books.

Bowlby, J. (1982). Attachment and loss: Vol. 1. Attachment (2nd ed.). New York: Basic Books. 
Bowlby, J. (1988). A secure base: Clinical applications of attachment theory. London: Routledge.

Bradbury, T. N., Fincham, F. D., \& Beach, S. R. H. (2000). Research on the nature and determinants of marital satisfaction: A decade in review. Journal of Marriage and the Family, 62, 964-980.

Brassard, A., Shaver, P. R., \& Lussier, Y. (2007). Attachment, sexual experience, and sexual pleasure in romantic relationships: A dyadic approach. Personal Relationships, 14, 475-493.

Brennan, K. A., Clark, C. L., \& Shaver, P. R. (1998). Self-report measurement of adult romantic attachment: An integrative overview. In J. A. Simpson \& W. S. Rholes (Eds.), Attachment theory and close relationships (pp. 46-76). New York: Guilford Press.

Butner, J., Diamond, L. M., \& Hicks, A. M. (2007). Attachment style and two forms of affect coregulation between romantic partners. Personal Relationships, 14, 431-455.

Call, V., Sprecher, S., \& Schwartz, P. (1995). The incidence and frequency of marital sex in a national sample. Journal of Marriage and the Family, 57, $639-652$.

Carnelley, K. B., Pietromonaco, P. R., \& Jaffe, K. (1994). Depression, working models of others, and relationship functioning. Journal of Personality and Social Psychology, 66, 127-140.

Carnelley, K. B., Pietromonaco, P. R., \& Jaffe, K. (1996). Attachment, caregiving, and relationship functioning in couples: Effects of self and partner. Personal Relationships, 3, 257-277.

Cassidy, J. (1994). Emotion regulation: Influences of attachment relationships. Monographs of the Society for Research in Child Development, 59, 228-283.

Cassidy, J., \& Shaver, P. R. (2008). Handbook of attachment: Theory, research, and clinical applications (2nd ed.). New York: Guilford Press.

Cavallo, J. V., Fitzsimons, G. M., \& Holmes, J. G. (2010). When self-protection overreaches: Relationship-specific threat activates domaingeneral avoidance motivation. Journal of Experimental Social Psychology, 46, $1-8$.

Ceglian, C. P., \& Gardner, S. (1999). Attachment style: A risk for multiple marriages? Journal of Divorce and Remarriage, 31, 125-139.

Cloutier P. F., Manion, I. G., Gordon Walker, J., \& Johnson, S. M. (2002). Emotionally focused interventions for couples with chronically ill children: A 2-year follow-up. Journal of Marital and Family Therapy, 28, 391-398.

Coan, J. A. (2008). Toward a neuroscience of attachment. In J. Cassidy \& P. R. Shaver (Eds.), Handbook of attachment: Theory, research, and clinical applications (2nd ed., pp. 241 -265). New York: Guilford Press.

Coan, J. A., Schaefer, H. S., \& Davidson, R. J. (2006). Lending a hand: Social regulation of the neural response to threat. Psychological Science, 17, $1032-1039$

Cobb, R. J., Davila, J., \& Bradbury, T. N. (2001). Attachment security and marital satisfaction: The role of positive perceptions and social support. Personality and Social Psychology Bulletin, 27, $1131-1143$.

Collins, N. L., \& Feeney, B. C. (2000). A safe haven: An attachment theory perspective on support seeking and caregiving in intimate relationships. Journal of Personality and Social Psychology, 78, $1053-1073$.

Cozzarelli, C., Hoekstra, S. J., \& Bylsma, W. H. (2000). General versus specific mental models of attachment: Are they associated with different outcomes? Personality and Social Psychology Bulletin, 26, 605-618.

Crowell, J. A., Fraley R. C., \& Shaver, P. R. (2008). Measurement of individual differences in adolescent and adult attachment. In J. Cassidy \& P. R. Shaver (Eds.), Handbook of attachment: Theory, research, and clinical applications (2nd ed., pp. 599-634). New York: Guilford Press.

Davila, J., \& Bradbury, T. N. (2001). Attachment insecurity and the distinction between unhappy spouses who do and do not divorce. Journal of Family Psychology, 15, 371-393.

Davila, J., Bradbury, T. N., \& Fincham, F. (1998). Negative affectivity as a mediator of the association between adult attachment and marital satisfaction. Personal Relationships, 5, 467-484.

Davila, J., Karney, B. R., \& Bradbury, T. N. (1999). Attachment change processes in the early years of marriage. Journal of Personality and Social Psychology, 76, 783-802.

Davila, J., \& Sargent, E. (2003). The meaning of life (events) predicts changes in attachment security. Personality and Social Psychology Bulletin, 29, $1383-1395$.

Depue, R. A., \& Morrone-Strupinsky, J. V. (2005). A neurobehavioral model of affiliative bonding: Implications for conceptualizing a human trait of affiliation. Behavioral and Brain Sciences, 28, $313-395$

Diamond, L. M. (2001). Contributions of psychophysiology to research on adult attachment: Review and recommendations. Personality and Social Psychology Review, 5, 276-295.

Diamond, L. M. (2003). What does sexual orientation orient? A biobehavioral model distinguishing romantic love and sexual desire. Psychological Review, 110, 173-192.

Diamond, L. M., \& Dubé, E. M. (2002). Friendship and attachment among heterosexual and sexualminority youths: Does the gender of your friend matter? Journal of Youth and Adolescence, 31, $155-166$.

Diamond, L. M., Hicks, A. M., \& Otter-Henderson, K. D. (2008). Everytime you go away: Changes in 
affect, behavior, and physiology associated with travel-related separations from romantic partners. Journal of Personality and Social Psychology, 95, $385-403$.

Dickerson, S. S., \& Kemeny, M. E. (2004). Acute stressors and cortisol responses: A theoretical integration and synthesis of laboratory research. Psychological Bulletin, 130, 355-391.

Ditzen, B., Neumann, I. D., Bodenmann, G., von Dawans, B., Turner, R. A., Ehlert, U., \& Heinrichs, M. (2007). Effects of different kinds of couple interaction on cortisol and heart rate responses to stress in women. Psychoneuroendocrinology, $32,565-574$.

Doherty, N. A., \& Feeney, J. A. (2004). The composition of attachment networks throughout the adult years. Personal Relationships, 11, 469-488.

Downey, G., Freitas, A. L., Michaelis, B., \& Khouri, H. (1998). The self-fulfilling prophecy in close relationships: Rejection sensitivity and rejection by romantic partners. Journal of Personality and Social Psychology, 75, 545-560.

Drigotas, S. M., Rusbult, C. E., Wieselquist, J., \& Whitton, S. (1999). Close partner as sculptor of the ideal self: Behavioral affirmation and the Michelangelo phenomenon. Journal of Personality and Social Psychology, 77, 293-323.

Dutton, D. G., Saunders, K., Starzomski, A., ～\& Bartholomew, K. (1994). Intimacy-anger and insecure attachment as precursors of abuse in intimate relationships. Journal of Applied Social Psychology, 24, $1367-1386$.

Ehlers, C. L., Frank, E., \& Kupfer, D. J. (1988). Social zeitgebers and biological rhythms: A unified approach to understanding the etiology of depression. Archives of General Psychiatry, 45, 948-952.

Elliot, A. J., \& Reis, H. T. (2003). Attachment and exploration in adulthood. Journal of Personality and Social Psychology, 85, 317-331.

Feeney, B. C. (2004). A secure base: Responsive support of goal strivings and exploration in adult intimate relationships. Journal of Personality and Social Psychology, 87, 631-648.

Feeney, B. C. (2007). The dependency paradox in close relationships: Accepting dependence promotes independence. Journal of Personality and Social Psychology, 92, 268-285.

Feeney, B. C., \& Thrush, R. L. (2010). Relationship influences in exploration in adulthood: The characteristics and functions of a secure base. Journal of Personality and Social Psychology, 98, 57-76.

Feeney, J. A. (1994). Attachment style, communication patterns, and satisfaction across the life cycle of marriage. Personal Relationships, 1, 333-348.

Feldman, R. (2007). Parent-infant synchrony: Biological foundations and developmental outcomes. Current Directions in Psychological Science, 16, $340-345$.
Fincham, F. D., \& Beach, S. R. H. (1999). Conflict in marriage: Implications for working with couples. Annual Review of Psychology, 50, 47-77.

Fraley, R. C., \& Brumbaugh, C. C. (2004). A dynamical systems approach to conceptualizing and studying stability and change in attachment security. In W. S. Rholes \& J. A. Simpson (Eds.), Adult attachment: Theory, research, and clinical implications (pp. 86-132). New York: Guilford Press.

Fraley, R. C., \& Davis, K. E. (1997). Attachment formation and transfer in young adults' close friendships and romantic relationships. Personal Relationships, 4, 131-144.

Fraley, R. C., \& Shaver, P. R. (1998). Airport separations: A naturalistic study of adult attachment dynamics in separating couples. Journal of Personality and Social Psychology, 75, 1198-1212.

Fraley, R. C., \& Spieker, S. J. (2003). Are infant attachment patterns continuously or categorically distributed? A taxometric analysis of strange situation behavior. Developmental Psychology, 39, $387-404$.

Fraley, R. C., \& Waller, N. G. (1998). Adult attachment patterns: A test of the typological model. In J. A. Simpson \& W. S. Rholes (Eds.), Attachment theory and close relationships (pp. 77-114). New York: Guilford Press.

Gable, S., Belsky, J., \& Crnic, K. (1992). Marriage, parenting, and child development: Progress and prospects. Journal of Family Psychology, 5, $276-294$.

Gentzler, A. L., \& Kerns, K. A. (2004). Associations between insecure attachment and sexual experiences. Personal Relationships, 11, 249-265.

Goodwin, R. (1999). Personal relationships across cultures. London: Routledge.

Gottman, J. M. (1982). Temporal form: Toward a new language for describing relationships. Journal of Marriage and the Family, 44, 943 - 962.

Gottman, J. M. (1994). What predicts divorce? The relationship between marital processes and marital outcomes. Hillsdale, NJ: Erlbaum.

Green, J. D., \& Campbell, W. (2000). Attachment and exploration in adults: Chronic and contextual accessibility. Personality and Social Psychology Bulletin, 26, 452-461.

Grewen, K. M., Anderson, B. J., Girdler, S. S., \& Light, K. C. (2003). Warm partner contact is related to lower cardiovascular reactivity. Behavioral Medicine, 29, 123-130.

Gump, B. B., Polk, D. E., Kamarck, T. W., \& Shiffman, S. M. (2001). Partner interactions are associated with reduced blood pressure in the natural environment: Ambulatory monitoring evidence from a healthy, multiethnic adult sample. Psychosomatic Medicine, 63, 423-433.

Hazan, C., Gur-Yaish, N., \& Campa, M. (2004). What does it mean to be attached? In W. S. Rholes \& J. A. Simpson (Eds.), Adult attachment: New 
directions and emerging issues (pp. 55-85). New York: Guilford Press.

Hazan, C., \& Shaver, P. R. (1987). Romantic love conceptualized as an attachment process. Journal of Personality and Social Psychology, 52, 511-524.

Hazan, C., \& Shaver, P. R. (1990). Love and work: An attachment-theoretical perspective. Journal of Personality and Social Psychology, 59, 270-280.

Hazan, C., \& Shaver, P. R. (1992). Broken attachments: Relationship loss from the perspective of attachment theory. In T. L. Orbuch (Ed.), Close relationship loss: Theoretical approaches (pp. 90-108). New York: Springer-Verlag.

Hazan, C., \& Shaver, P. R. (1994). Attachment as an organizational framework for research on close relationships. Psychological Inquiry, 5, 1-22.

Hazan, C., \& Zeifman, D. (1994). Sex and the psychological tether. In K. Bartholomew \& D. Perlman (Eds.), Advances in personal relationships: Attachment processes in adulthood (Vol. 5, pp. $151-177)$. London: Jessica Kingsley.

Hill, E. M., Young, J. P., \& Nord, J. L. (1994). Childhood adversity, attachment security, and adult relationships: A preliminary study. Ethology and Sociobiology, 15, 323-338.

Hofer, M. A. (1994). Hidden regulators in attachment, separation, and loss. Monographs of the Society for Research in Child Development, 59, 250-283.

Holt-Lunstad, J., Uchino, B. N., Smith, T. W., OlsonCerny, C., \& Nealey-Moore, J. B. (2003). Social relationships and ambulatory blood pressure: Structural and qualitative predictors of cardiovascular function during everyday social interactions. Health Psychology, 22, 388-397.

Holtzworth-Munroe, A., Stuart, G. L., \& Hutchinson, G. (1997). Violent versus nonviolent husbands: Differences in attachment patterns, dependency, and jealousy. Journal of Family Psychology, 11, 314-331.

Irwin, M., Hauger, R., \& Brown, M. (1992). Central corticotropin-releasing hormone activates the sympathetic nervous system and reduces immune function: Increased responsivity of the aged rat. Endocrinology, 131, 1047-1053.

Jang, S. A., Smith, S. W. \& Levine, T. R. (2002). To stay or to leave? The role of attachment styles in communication patterns and potential termination of romantic relationships following discovery of deception. Communication Monographs, 69, 236- 252 .

Johnson, S. M. (2004). Attachment theory: A guide for healing couple relationships. In W. S. Rholes \& J. A. Simpson (Eds.), Adult attachment: New directions and emerging issues (pp. 367-387). New York: Guilford Press.

Johnson, S. M., Hunsley, J., Greenberg, L., \& Schindler, D. (1999). Emotionally focused couples therapy: Status and challenges. Clinical Psychology: Science and Practice, 6, 67-79.
Kamarck, T., \& Jennings, J. R. (1991). Biobehavioral factors in sudden cardiac death. Psychological Bulletin, 109, 42-75.

Karney, B. R., \& Bradbury, T. N. (1995). The longitudinal course of marital quality and stability: A review of theory, method, and research. Psychological Bulletin, 118, 3-34.

Kitson, G. C., \& Raschke, H. J. (1981). Divorce research: What we know, what we need to know. Journal of Divorce, 4, 1-37.

Klohnen, E. C., \& Bera, S. (1998). Behavioral and experiential patterns of avoidantly and securely attached women across adulthood: A 31-year longitudinal perspective. Journal of Personality and Social Psychology, 74, 211-223.

Kumashiro, M., Rusbult, C. E., Wolff, S. T., Estrada, M. J. (2006). The Michelangelo phenomenon: Partner affirmation and self-movement toward one's ideal. In K. D. Vohs \& E. J. Finkel (Eds.), Self and relationships: Connecting intrapersonal and interpersonal processes (pp. 317-341). New York: Guilford Press.

Mackey, R. A., Diemer, M. A., \& O’Brien, B. A. (2004). Relational factors in understanding satisfaction in the lasting relationships of same-sex and heterosexual couples. Journal of Homosexuality, 47, $111-136$.

Main, M., \& Hesse, E. (1990). Parents' unresolved traumatic experiences are related to infant disorganized attachment status: Is frightened and/or frightening parental behavior the linking mechanism? In M. T. Greenberg, D. Cicchetti, \& E. M. Cummings (Eds.), Attachment in the preschool years: Theory, research, and intervention (pp. 161-182). Chicago: University of Chicago Press.

Marchand, J. F. (2004). Husbands' and wives' marital quality: The role of adult attachment orientations, depressive symptoms, and conflict resolution behaviors. Attachment and Human Development, 6, 99-112.

Mason, W. A., \& Mendoza, S. P. (1998). Generic aspects of primate attachments: Parents, offsprings, and mates, Psychoneuroendocrinology, 23, $765-778$.

Master, S. L., Eisenberger, N. I., Taylor, S. E., Naliboff, B. D., Shirinyan, D., \& Lieberman, M. D. (2009). A picture's worth: Partner photographs reduce experimentally induced pain. Psychological Science, 20, 1316-1318.

McClure, E. B. (2000). A meta-analytic review of sex differences in facial expression processing and their development in infants, children, and adolescents. Psychological Bulletin, 126, 424-453.

McCubbin, J. A. (1993). Stress and endogenous opioids: Behavioral and circulatory interactions. Biological Psychology, 35, 91-122.

McEwen, B. S., \& Stellar, E. (1993). Stress and the individual: Mechanisms leading to disease. Archives of Internal Medicine, 153, 2093-2101. 
Mikulincer, M., Birnbaum, G., Woddis, D., \& Nachmias, O. (2000). Stress and accessibility of proximity-related thoughts: Exploring the normative and intraindividual components of attachment theory. Journal of Personality and Social Psychology, 78, 509-523.

Mikulincer, M., Florian, V., Birnbaum, G., \& Malishkevich, S. (2002). The death-anxiety buffering function of close relationships: Exploring the effects of separation reminders on death-thought accessibility. Personality and Social Psychology Bulletin, 28, 287-299.

Mikulincer, M., \& Shaver, P. R. (2004). Securitybased self-representations in adulthood: Contents and processes. In W. S. Rholes \& J. A. Simpson (Eds.), Adult attachment: Theory, research, and clinical implications (pp. 159-195). New York: Guilford Press.

Mikulincer, M., \& Shaver, P. R. (2007). Attachment in adulthood: Structure, dynamics, and change. New York: Guilford Press.

Mohr, J. J. (2008). Same-sex romantic attachment. In J. Cassidy \& P. R. Shaver (Eds.), Handbook of attachment: Theory, research, and clinical applications (2nd ed., pp. 482-502). New York: Guilford Press.

Neff, L. A., \& Karney, B. R. (2005). Gender differences in social support: A question of skill or responsiveness? Journal of Personality and Social Psychology, 88, 79-90.

Parkes, C. M. (2006). Love and loss: The roots of grief and its complications. New York: Taylor and Francis.

Parkes, C. M., \& Weiss, R. S. (1983). Recovery from bereavement. New York: Basic Books.

Polan, J. H., \& Hofer, M. A. (2008). Psychobiological origins of infant attachment and its role in development. In J. Cassidy \& P. R. Shaver (Eds.), Handbook of attachment: Theory, research, and clinical applications (2nd ed., pp. $158-172$ ). New York: Guilford Press.

Rankin, L. B., Saunders, D. G., \& Williams, R. A. (2000). Mediators of attachment style, social support, and sense of belonging in predicting woman abuse by African American men. Journal of Interpersonal Violence, 15, 1060-1080.

Rholes, W. S., Simpson, J. A., \& Friedman, M. (2006). Avoidant attachment and the experience of parenting. Personality and Social Psychology Bulletin, 32, 275-285.

Rholes, W. S., Simpson, J. A., \& Oriña, M. M. (1999). Attachment and anger in an anxiety-provoking situation. Journal of Personality and Social Psychology, 76, 940-957.

Robles, T. F., \& Kiecolt-Glaser, J. K. (2003). The physiology of marriage: pathways to health. Physiology and Behavior, 79, 409-416.

Rosenblatt, P., \& Anderson, R. (1981). Human sexuality in cross-cultural perspective. In M. Cook
(Ed.), The bases of human sexual attraction (pp. 215-250). New York: Academic Press.

Sampson, R. J., Laub, J. H., \& Wimer, C. (2006). Does marriage reduce crime? A counterfactual approach to within-individual causal effects. Criminology, 44, 465-508.

Saxbe, D. E., \& Repetti, R. L. (2010). For better or worse? Coregulation of couples' cortisol levels and mood states. Journal of Personality and Social Psychology, 98, 92-103.

Saxbe, D. E., Repetti, R. L., \& Nishina, A. (2008). Marital satisfaction, recovery from work, and diurnal cortisol among men and women. Health Psychology, 27, 15-25.

Sbarra, D. A., \& Hazan, C. (2008). Coregulation, dysregulation, self-regulation: An integrative analysis and empirical agenda for understanding adult attachment, separation, loss, and recovery. Personality and Social Psychology Review, 12, $141-167$.

Scharfe, E., \& Bartholomew, K. (1994). Reliability and stability of adult attachment patterns. Personal Relationships, 1, 23-43.

Schmitt, D. P., Alcalay, L., Allensworth, M., Allik J, Ault, L., Austers, I. et al. (2003). Are men universally more dismissing than women? Gender differences in romantic attachment across 62 cultures. Personal Relationships, 10, 307-331.

Schmitt, D. P., Alcalay, L., Allensworth, M., Allik J, Ault, L., Austers, I. et al. (2004). Patterns and universals of adult romantic attachment across 62 cultures: Are models of self and other pancultural constructs? Journal of Cross-Cultural Psychology, $35,367-402$.

Selcuk, E., \& Hazan, C. (2010). Attachment and exploration: Priming romantic partners increases curiosity and exploration. Unpublished manuscript, Cornell University.

Senchak, M., \& Leonard, K. E. (1992). Attachment styles and marital adjustment among newlywed couples. Journal of Social and Personal Relationships, 9, 51-64.

Shaver, P. R., Schachner, D. A., \& Mikulincer, M. (2005). Attachment style, excessive reassurance seeking, relationship processes, and depression. Personality and Social Psychology Bulletin, 31, $343-359$.

Sroufe, L. A., \& Waters, E. (1977a). Attachment as an organizational construct. Child Development, 48, 1184-1199.

Sroufe, L. A., \& Waters, E. (1977b). Heart rate as a convergent measure in clinical and developmental research. Merrill-Palmer Quarterly, 23, 3-27.

Thayer, J. F., Friedman, B. H., \& Borkovec, T. D. (1996). Autonomic characteristics of generalized anxiety disorder and worry. Biological Psychiatry, 39, 255-266.

Tran, S., \& Simpson, J. A. (2009). Prorelationship maintenance behaviors: The joint roles of 
attachment and commitment. Journal of Personality and Social Psychology, 97, 685-698.

Trinke, S. J., \& Bartholomew, K. (1997). Hierarchies of attachment relationships in young adulthood. Journal of Social and Personal Relationships, 14, 603-625.

U.S. Census Bureau. (2000). Equal employment opportunity. Retrieved from http://www.census. gov/hhes/www/eeoindex/page_c.html

Uvnäs-Moberg, K. (1998). Oxytocin may mediate the benefits of positive social interaction and emotions. Psychoneuroendocrinology, 23, 819-835.

van IJzendoorn, M. H., \& Sagi-Schwartz, A. (2008). Cross-cultural patterns of attachment: Universal and contextual dimensions. In J. Cassidy \& P. R. Shaver (Eds.), Handbook of attachment: Theory, research, and clinical applications (2nd ed., pp. 880-905). New York: Guilford Press.

Vigil, J. M. (2009). A socio-relational framework of sex differences in the expression of emotion. Behavioral and Brain Sciences, 32, 375-390.
Volling, B. L., Notaro, P. C., \& Larsen, J. J. (1998). Adult attachment styles: Relations with emotional well-being, marriage, and parenting. Family Relations, 47, 355-367.

Vormbrock, J. K. (1993). Attachment theory as applied to wartime and job-related marital separation. Psychological Bulletin, 114, 122 - 144.

Weiss, R. S. (1975). Marital separation. New York: Basic Books.

Young, L. J., \& Wang, Z. (2004). The neurobiology of pair bonding. Nature Neuroscience, 10, $1048-1054$.

Wynne, L. C. (1988). An epigenetic model of family processes. In C. J. Falicov (Ed.), Family transitions: Continuity and change over the life cycle (pp. 81-106). New York: Guilford Press.

Zeifman, D., \& Hazan, C. (2008). Pair bonds as attachments: Reevaluating the evidence. In J. Cassidy \& P. R. Shaver (Eds.), Handbook of attachment: Theory, research, and clinical applications (2nd ed., pp. 436-455). New York: Guilford Press. 